\title{
Fungal deterioration of the bagasse storage from the harvested sugarcane
}

\author{
Na Peng ${ }^{1 \dagger}$, Ziting Yao ${ }^{1+}$, Ziting Wang ${ }^{1}$, Jiangfeng Huang ${ }^{1}$, Muhammad Tahir Khan ${ }^{2}$, Baoshan Chen ${ }^{1}$ and \\ Muqing Zhang ${ }^{1 *}$ (D)
}

\begin{abstract}
Background: Sugarcane is an essential crop for sugar and ethanol production. Immediate processing of sugarcane is necessary after harvested because of rapid sucrose losses and deterioration of stalks. This study was conducted to fill the knowledge gap regarding the exploration of fungal communities in harvested deteriorating sugarcane. Experiments were performed on simulating production at $30^{\circ} \mathrm{C}$ and $40^{\circ} \mathrm{C}$ after 0,12 , and $60 \mathrm{~h}$ of sugarcane harvesting and powder-processing.

Results: Both $\mathrm{pH}$ and sucrose content declined significantly within $12 \mathrm{~h}$. Fungal taxa were unraveled using ITS amplicon sequencing. With the increasing temperature, the diversity of the fungal community decreased over time. The fungal community structure significantly changed within $12 \mathrm{~h}$ of bagasse storage. Before stored, the dominant genus (species) in bagasse was Wickerhamomyces (W. anomalus). Following storage, Kazachstania (K. humilis) and Saccharomyces (S. cerevisiae) gradually grew, becoming abundant fungi at $30^{\circ} \mathrm{C}$ and $40^{\circ} \mathrm{C}$. The bagasse at different temperatures had a similar pattern after storage for the same intervals, indicating that the temperature was the primary cause for the variation of core features. Moreover, most of the top fungal genera were significantly correlated with environmental factors ( $\mathrm{pH}$ and sucrose of sugarcane, storage time, and temperature). In addition, the impact of dominant fungal species isolated from the deteriorating sugarcane on sucrose content and $\mathrm{pH}$ in the stored sugarcane juice was verified.
\end{abstract}

Conclusions: The study highlighted the importance of timeliness to refine sugar as soon as possible after harvesting the sugarcane. The lessons learned from this research are vital for sugarcane growers and the sugar industry for minimizing post-harvest losses.

Keywords: Sugarcane biodegradation, Post-harvest storing, Fungal community, Yeast, Sucrose losses

\section{Background}

Sugarcane is one of the most crucial crops for sugar and bioethanol production [1]. The estimated annual gross sugar output from sugarcane is valued as high as $\$ 76$ billion [2]. Sugarcane is a perishable commodity and must

*Correspondence: zmuqing@163.com

${ }^{\dagger} \mathrm{Na}$ Peng and Ziting Yao contributed equally to this paper

${ }^{1}$ Guangxi Key Laboratory for Sugarcane Biology \& State Key Laboratory

for Conservation and Utilization of Subtropical Agro-Bioresources,

Guangxi University, Nanning 530005, China

Full list of author information is available at the end of the article be processed into sugar quickly after harvested. The postharvest deterioration of sugarcane is a severe problem for the sugar industry, causing 20-30 percent sucrose losses in cane-producing countries [2-5]. Many factors are associated with the deterioration of the harvested cane, including cane cultivar and its maturity, mechanical or manual harvesting, exposure to microbes, cut-to-crush delay, and storage $[2,5,6]$.

The sugar industry prefers pre-harvest sugarcane burning during sugarcane harvesting globally because it reduces transportation costs and makes the harvest process quicker and more accessible $[7,8]$. In many 
countries, the harvested cane is kept in the field for 3-5 days due to the flawed field transport system and 1-3 days in the factory storage under undesirable conditions $[2,5]$. Sucrose inversions in the deterioration of harvested cane result from the chemical (acid), enzymic inversion, and microbial activity. In the first $14 \mathrm{~h}$ of cane juice deterioration, $93.0 \%$ of sucrose losses are caused by microbial, $5.7 \%$ by enzymic, and $1.3 \%$ by chemical changes (acid degradation) [3]. Microorganism plays a significant role in biodegradation and changes of host chemical characteristics $[9,10]$. The high sugar concentration within the mature internodes provides a favorable environment for microbial thriving, which enters the harvested stalk through wounds or cut ends [6]. Also, leaf sheaths and growth cracks provide excellent sites for microbial growth. Over 400 species of bacteria and fungi are associated with sugarcane products [11]. After milling, Penicillium, Lactobacillus, Leuconostoc, and yeast invade the stored sugarcane $[2,12,13]$. These acid-producing microorganisms cause deterioration, decreasing sucrose content, juice purity, and $\mathrm{pH}$, especially under anaerobic conditions such as mud-coated canes and the cane stored in large piles with poor ventilation $[5,13]$. The glucose and fructose are converted to organic acids and mannitol by the enzymes secreted by these microorganisms. Besides these external microbes, the endophytic microbial genera viz., Acetobacter, Enterobacter, Pseudomonas, Aeromonas, Vibrio, Bacillus, and lactic acid group are also responsible for the deterioration of juice quality during staling $[13,14]$.

It is crucial to identify the key players in sugarcane deterioration and improve early detection strategies of degradation-causing microbes. The sucrose-related dynamics of fungi in stored sugarcane bagasse remain unexplored. Thereby, the fungal community variations in the stored bagasse were analyzed for assessing the core fungi and specific biodegradation biomarkers using high-throughput sequencing. Moreover, the study also explored the impact of different cane storage durations and temperatures on sugarcane deterioration and dissected these parameters associated with the fungal richness and diversity.

\section{Results}

\section{Sucrose and $\mathrm{pH}$}

In the first $12 \mathrm{~h}$, the steepest decline was observed in the bagasse stored at $30{ }^{\circ} \mathrm{C}$. The sucrose reduced by $53.9 \%$ within the first $12 \mathrm{~h}$ and then completely decomposed during the initial $36 \mathrm{~h}$ for all the treated bagasse. Contrary to sucrose, the glucose and fructose of the bagasse increased in the first $12 \mathrm{~h}$ and then fell to zero by $60 \mathrm{~h}$ (Fig. 1b, c). However, the glucose and fructose increased in the bagasse stored at $40{ }^{\circ} \mathrm{C}$ was higher than those at
$30{ }^{\circ} \mathrm{C}$. The $\mathrm{pH}$ of all the bagasse reduced significantly in the first $12 \mathrm{~h}$ (one-way ANOVA, $p<0.001$ ) and then maintained at around 3.6-3.8 (Fig. 1).

\section{Diversity of fungal communities}

For determining the fungi prevailing in biodegrading sugarcane, microbial community DNA was extracted and amplified using the ITS2 primer pair (Additional file 1: Fig. S3). Subsequently, the amplicons were sequenced on the Illumina MiSeq platform. A total of 962,640 reads were recovered from all samples after quality filtering (Additional file 1: Tables S1 and S2), representing 31 fungal Operational Taxonomic Units (OTUs) at 97\% sequence similarity. The rarefaction curves confirmed that all the samples reached the plateau phase (Additional file 1: Fig. S1). The richness and diversity of fungal OTUs rose in the first $12 \mathrm{~h}$ in all the samples. The fungal richness and diversity were significantly lower in the bagasse stored at $40{ }^{\circ} \mathrm{C}$ for $60 \mathrm{~h}$ than those stored at $30{ }^{\circ} \mathrm{C}(p \leq 0.05)$ (Fig. 2). Principal component analysis was used for the OUT data obtained from the 12 treatments and three pre-treatments (CK) after standardization as described in the Methods. The first principal component accounted for $45 \%$ of the total variance in the data set, while the second principal component explained $22 \%$. Bagasse stored at $30{ }^{\circ} \mathrm{C}$ and $40{ }^{\circ} \mathrm{C}$ were quite different from the pretreated bagasse (CK) (Fig. 3). The storage temperature alone explained $34.7 \%$ of the microbial community variation (PERMANOVA, $p=0.020$ ), while the storage duration explained $73.7 \%$ of the variation (PERMANOVA, $p<0.001$ ).

Only Ascomycota was identified at the phylum level. Four classes were identified at the class level, including Saccharomycetes, Dothideomycetes, Sordariomycetes, and Eurotiomycetes, of which Saccharomycetes accounted for 99.7\%. Five orders were identified, including Saccharomycetales, Pleosporales, Hypocreales, Chaetothyriales, and Eurotiales, of which Saccharomycetales alone accounted for $99.7 \%$. At the family level, ten families were identified, of which Saccharomycetaceae accounted for $64.1 \%$, followed by Phaffomycetaceae (35.3\%). Further, at the genus level, a total of 14 fungal genera were identified. Saccharomyces, Torulaspora, Hanseniaspora, and Curvularia were exclusively present in the bagasse stored at $40{ }^{\circ} \mathrm{C}$, while Kazachstania, Zygosaccharomyces, and Hanseniaspora were solely identified in bagasse stored at $30^{\circ} \mathrm{C}$. The dominant genus in CK bagasse was Wickerhamomyces (92\%).

When the bagasses were stored at $30{ }^{\circ} \mathrm{C}$, Kazachstania gradually replaced Wickerhamomyces as the most abundant fungus $(60 \%$ at $12 \mathrm{~h}$ and $80 \%$ at $60 \mathrm{~h}$ ). However, the bagasses stored at $40{ }^{\circ} \mathrm{C}$ for $12 \mathrm{~h}$ had two highly abundant genera of Saccharomyces (50\%) and 

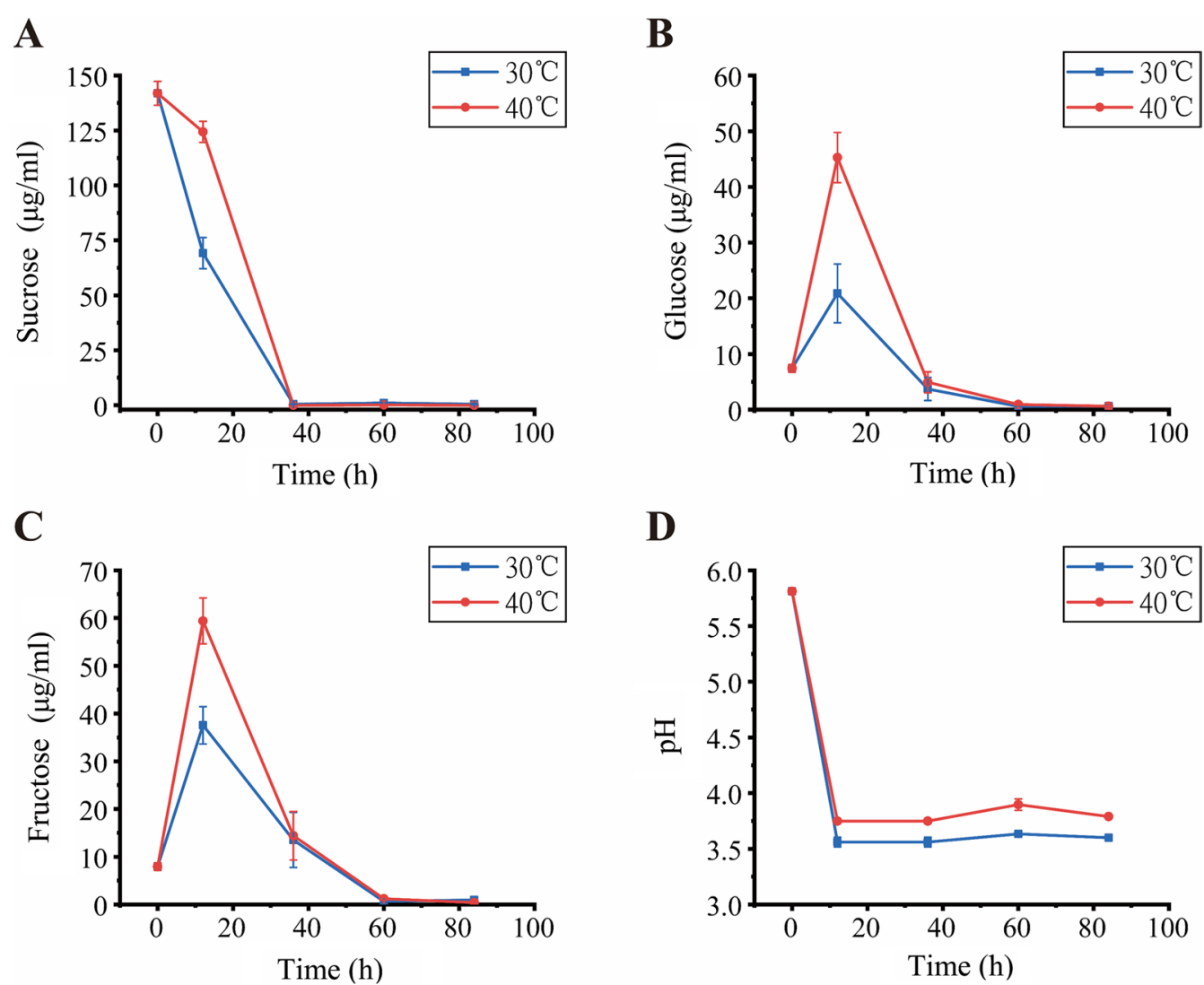

Fig. 1 Properties of sugarcane stem samples. Sucrose (A), glucose (B), fructose $(\mathbf{C})$, and pH (D) profiles in the stem powder recorded during the storage period. Data were presented as the mean \pm standard deviation

Wickerhamomyces (50\%). The relative abundance of Saccharomyces increased up to $90 \%$ after $60 \mathrm{~h}$ in these bagasses (Fig. 4a). At the species level, both Saccharomyces (S. cerevisiae) or non-Saccharomyces yeast (K. humilis and $W$. anomalous) were predominant and varied with the increase in temperature and duration of the storage. Before temperature treatment (CK), W. anomalous accounted for $92.4 \%$ of all tested OTUs. When stored at $30{ }^{\circ} \mathrm{C}, \mathrm{K}$. humilis was predominant, and its abundance increased from 3.5\% (before treatment) to $68.8 \%$ after $12 \mathrm{~h}$ and $82.9 \%$ after $60 \mathrm{~h}$. On the other hand, $W$. anomalus sharply decreased to $8.6 \%$ after $60 \mathrm{~h}$ of treatment, while $S$. cerevisiae increased to $7.2 \%$. S. cerevisiae dominated at $40{ }^{\circ} \mathrm{C}$, accounting for $49.9 \%$ after $12 \mathrm{~h}$ and $99.6 \%$ after $60 \mathrm{~h}$, whereas $W$. anomalous sharply reduced from $44.8 \%$ after $12 \mathrm{~h}$ to an undetectable level after $60 \mathrm{~h}$. Only $2.4 \%$ of $K$. humilis was detected at $40{ }^{\circ} \mathrm{C}$ after $12 \mathrm{~h}$ of storage (Fig. 4b).

\section{The primary driver for fungal community composition}

The redundancy analysis (RDA) was used to determine how the environmental parameters affected the fungal community composition (Fig. 5). Among environmental factors, sucrose content $(F$-ratio $=77.53$, $p=0.002)$ and storage time $(F$-ratio $=65.5, p=0.003)$ are significantly associated with the fungal communities. The storage temperature $(F$-ratio $=30.67)$ and $\mathrm{pH}$ $(F$-ratio $=37.33)$ also played an essential role in the variation of fungal communities. Hence, RDA showed that the fungal communities varied with the environmental factors.

The correlation heatmap of the relationships among the top 14 genera and six selected environmental factors (storage temperature, storage time, sucrose content, fructose content, glucose content, and $\mathrm{pH}$ ) is presented in Fig. 6. The storage temperature was significantly negatively correlated with $\mathrm{pH}$, i.e., the higher the storage temperature, the lower the $\mathrm{pH}$. This association supported the higher growth of Saccharomyces and negatively impacted the richness of any other fungal genera. The storage time had a significant influence on the conversion of sucrose to glucose and fructose. Fructose and glucose content slightly correlated with the fungal abundance, while sucrose significantly affected Wickerhamomyces and Torulaspora. 

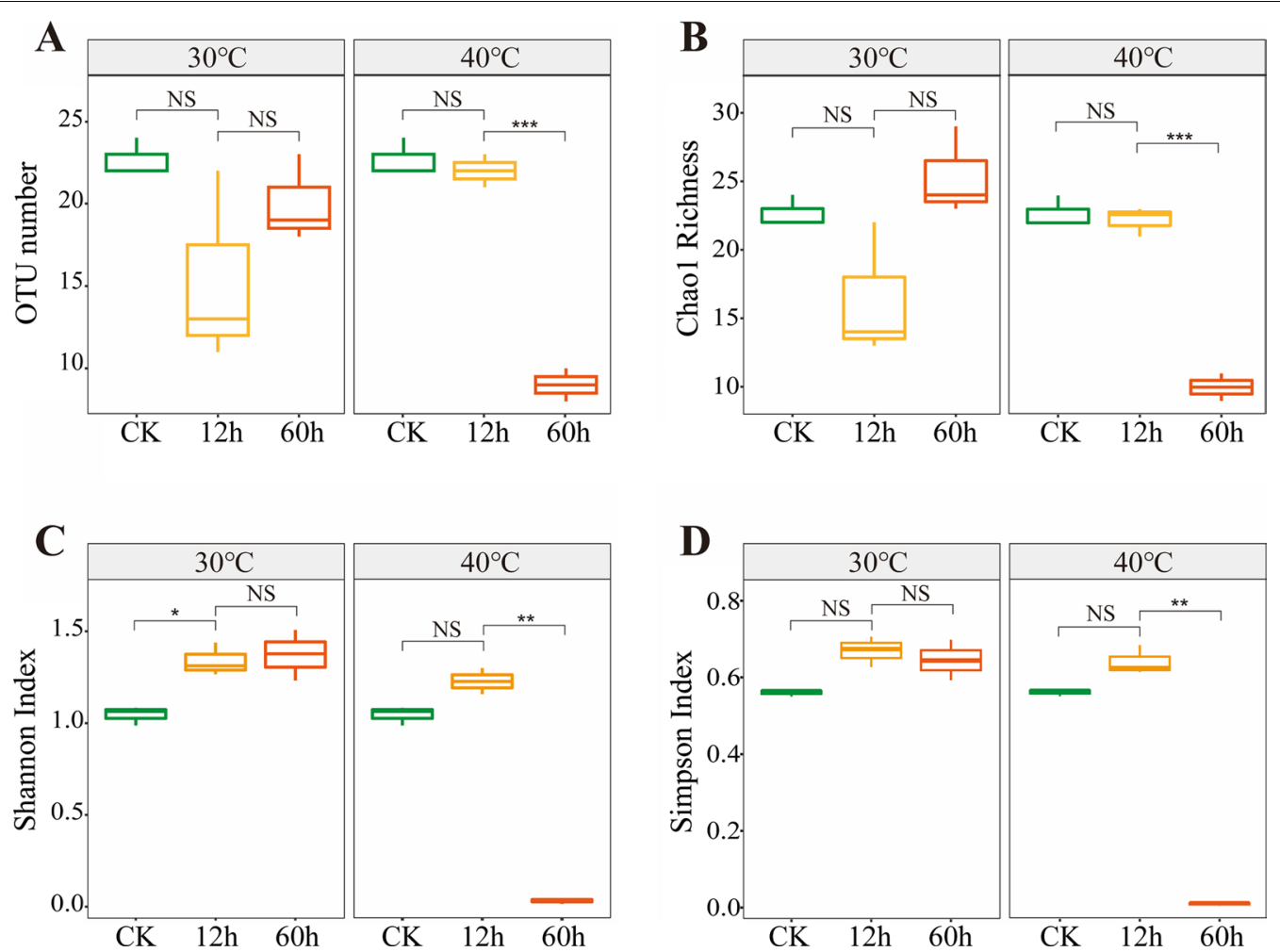

Fig. 2 Sequences rarefaction curve and fungal diversity of sugarcane stem samples. Observed OTUs numbers (A), Chao richness index (B), Shannon diversity index $(\mathbf{C})$, and Simpson diversity index (D). Each sample was determined in triplicate $(n=3)$. Data were shown as average \pm SD. ${ }^{*}$ Indicates significant correlation at $p<0.05,{ }^{* *}$ indicated significant correlation at $p<0.01$, and ${ }^{* * *}$ indicated significant correlation at $p<0.001$

\section{Functional verification of isolated strains}

Five fungal genera were isolated by plate culture. Further, the utilization of sucrose was assessed by inoculating fungal solutions in the sucrose medium. Saccharomyces, Wickerhamomyces, and Torulaspora had a solid ability to degrade sucrose at $30^{\circ} \mathrm{C}$, consuming sucrose entirely within $36 \mathrm{~h}$ (Fig. 7). This phenomenon also yielded different reducing sugar byproducts in different quantifies (Additional file 1: Fig. S2). However, at $40{ }^{\circ} \mathrm{C}$, only Saccharomyces maintained the same activity, and the sucrose in its culture medium was completely degraded within $36 \mathrm{~h}$. All other fungi were sensitive to temperature. Consistent with the sucrose contents, the $\mathrm{pH}$ dropped in the media culturing the strains. At $30{ }^{\circ} \mathrm{C}$, the $\mathrm{pH}$ dropped to 3.6-3.8 after $36 \mathrm{~h}$. The $\mathrm{pH}$ of the media having Saccharomyces and Wickerhamomyces reduced faster (Fig. 8). At $40{ }^{\circ} \mathrm{C}$, only the $\mathrm{pH}$ decreased significantly in the medium inoculated with Saccharomyces.

\section{Discussion}

The severity of sugarcane deterioration is influenced by many factors, including the length of the harvest-tocrush delay, ambient temperature, and harvesting practices. Pre-harvest burning cane exacerbates post-harvest deterioration rates and requires a short delay of the harvest-to-crush, with a burnt cane left more exposed and reaching higher deterioration levels than green cane $[15$, 16]. Sugarcane staling is one of the significant issues of the sugar industry, and it causes significant economic losses because of cane and sucrose deterioration during storage and transportation [5, 17]. The post-harvest sugarcane biodegradation necessitates the abating of harvest-to-crush delays. Microbial communities thriving the harvested sugarcane play a crucial role in this deterioration. Moreover, other factors, such as storage time, temperature, and environmental conditions, are essential, too $[2,5,6]$. As per our knowledge, the sucrose-related dynamics of fungi in stored sugarcane remain unexplored. Therefore, this study was conducted to investigate the diversity and abundance of fungal communities in harvested sugarcane. Further, we also examined the impact of different storage temperatures and duration on deterioration processes and fungal abundance.

The $\mathrm{pH}$ and sucrose sharply reduced in the first $12 \mathrm{~h}$ and then remained stable over $36 \mathrm{~h}$. However, both fructose and glucose increased in the first $12 \mathrm{~h}$, followed by a sharp decrease. The richness and diversity of fungal OTUs declined with the increase in storage 


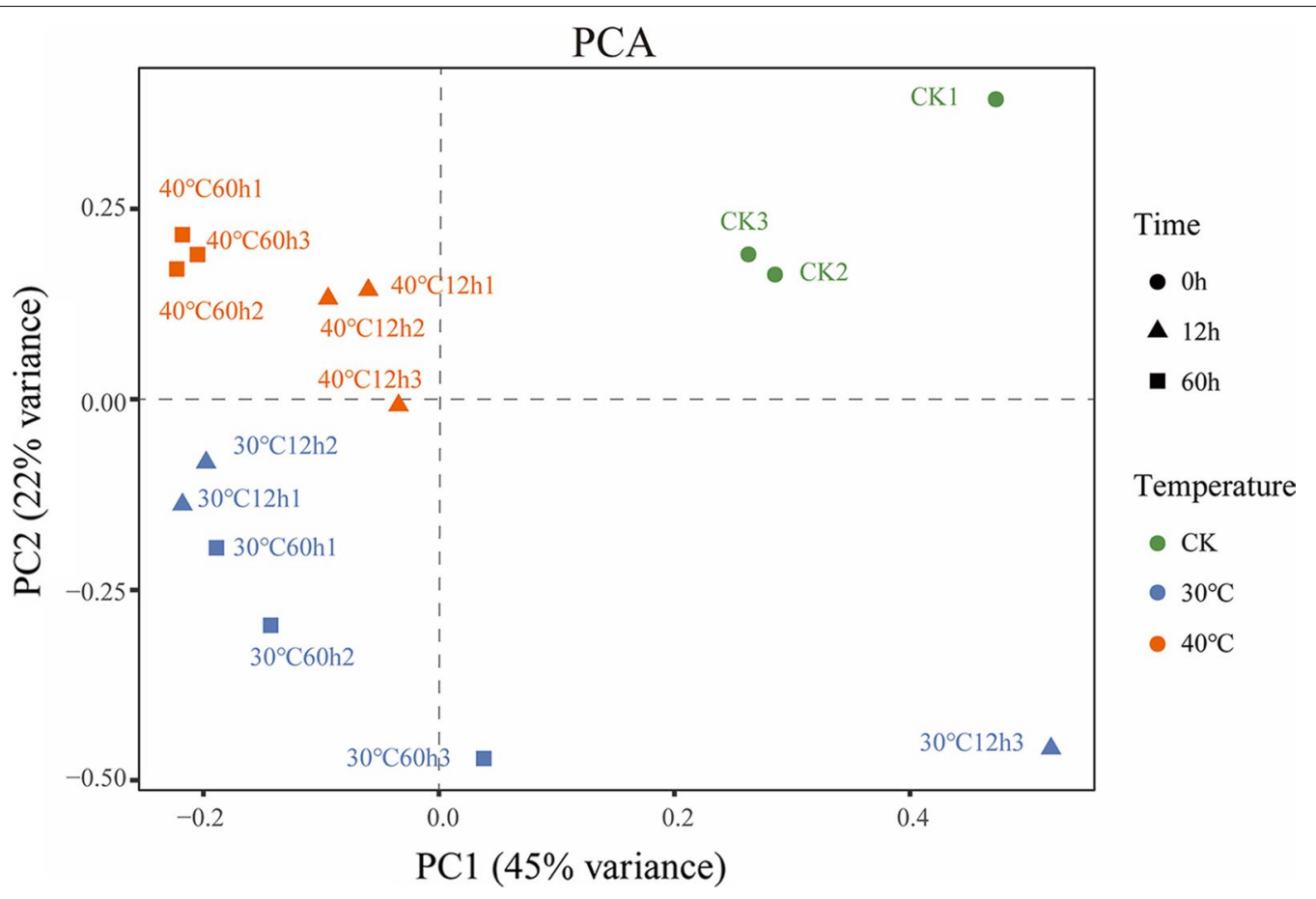

Fig. 3 Fungal community structure in sugarcane stem samples. The figure presented principal coordinate analysis (PCoA) of ITS2 sequences diversity in the stored stem at the disparate temperatures used in the study. The different colors indicated the different temperatures of the storage and fungal communities from the sugarcane stem

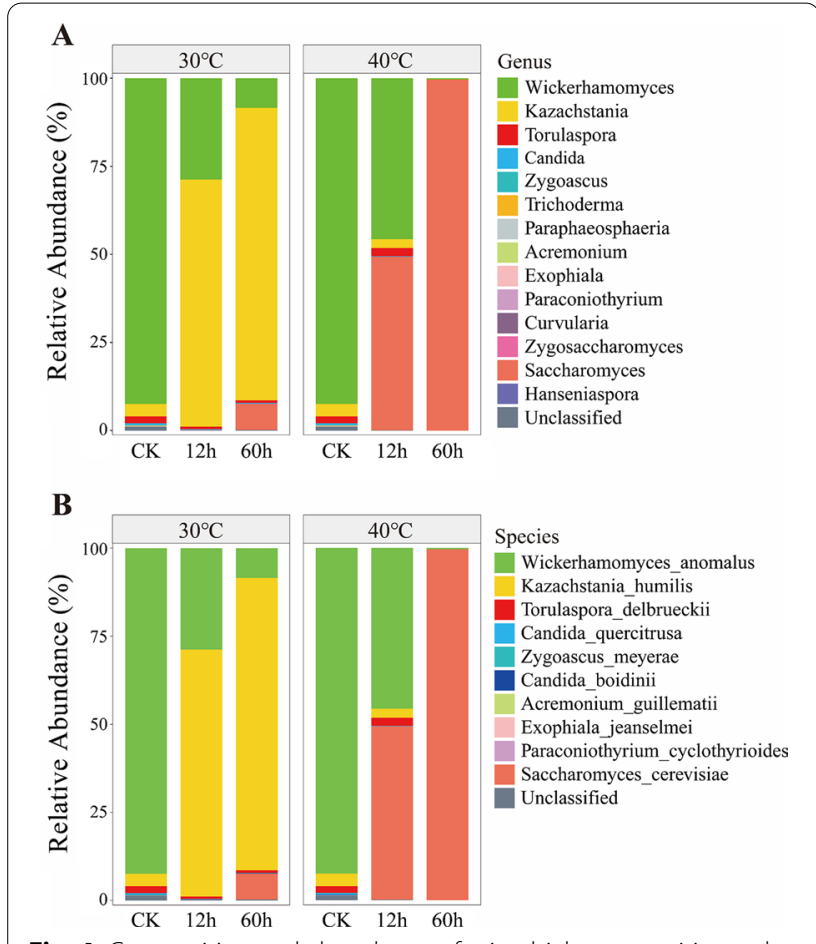

Fig. 4 Composition and abundance of microbial communities at the genus (A) and species (B)

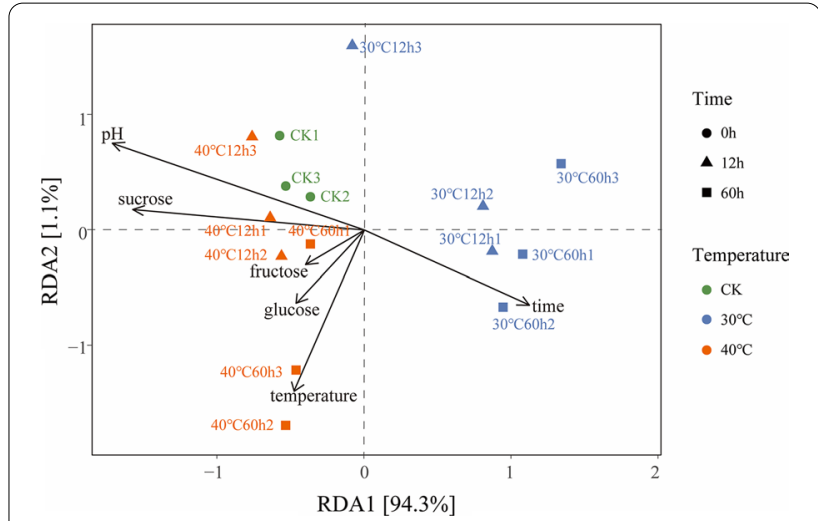

Fig. 5 Redundancy analysis (RDA) for the correlation between the fungal community and environmental factors

temperature and duration. Wickerhamomyces (W. anomalus), Kazachstania (K. humilis), and Saccharomyces ( $S$. cerevisiae) were the most abundant fungal genera (species) in the deterioration of harvested sugarcane. S. cerevisiae dominated in the bagasse stored at $40{ }^{\circ} \mathrm{C}$, ranging from $49.9 \%$ at $12 \mathrm{~h}$ to $99.6 \%$ at $60 \mathrm{~h}$. At $30{ }^{\circ} \mathrm{C}, \mathrm{K}$. humilis enriched from $3.5 \%$ in pretreated bagasse to $82.9 \%$ at $60 \mathrm{~h}$ post-treatment. W. anomalus was more prevalent in the pretreated bagasse, 


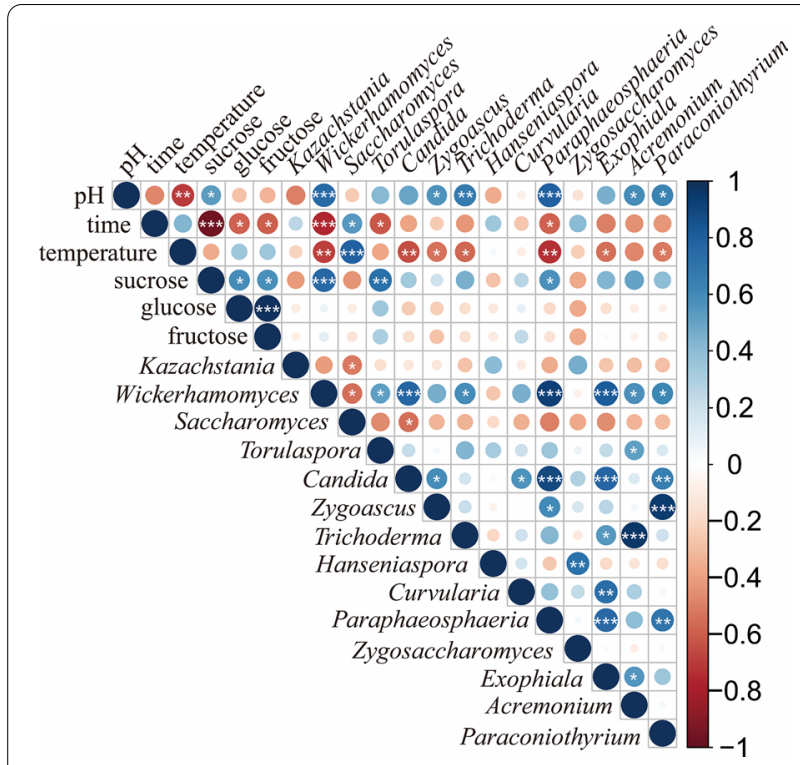

Fig. 6 The correlation heatmap of the relationships among the top 14 genera and six selected environmental factors. The minus value of Spearman's correlation index denoted a negative correlation, while a positive value denoted a positive correlation. ${ }^{*}$ Indicated significant correlation at $p<0.05,{ }^{* *}$ indicated significant correlation at $p<0.01$, and *** indicated significantly correlation at $p<0.001$

decreasing from 92.4 to $8.6 \%$ at $30{ }^{\circ} \mathrm{C}$ and an undetectable level at $40{ }^{\circ} \mathrm{C}$ after $60 \mathrm{~h}$.

Wickerhamomyces anomalus, with wide metabolic and physiological diversity, assimilates sucrose, lactose, and starch for its growth [18, 19]. As a classical killer yeast, $W$. anomalus has an antifungal activity to produce protein toxins or low molecular mass glycoproteins lethal to other susceptible yeasts and filamentous fungi (Aspergillus, Botrytis, Penicillium, Fusarium) [20-26]. The antifungal properties of $W$. anomalus could be the reason that richness and diversity were very low in our bagasse samples. Only 31 OTUs were assembled from 962,640 reads of our high-throughput sequencing. $W$. anomalus is sensitive to oxygen, whose respiratory growth is favored under aerobic conditions. At the same time, alcoholic fermentation is induced under limited oxygen conditions $[19,27]$. W. anomalus exhibited growth rates of 0.22 and $0.056 \mathrm{~h}^{-1}$ and biomass yields of 0.59 and $0.11 \mathrm{~g} / \mathrm{g}$ glucose under aerobic and anaerobic conditions, respectively [28]. Therefore, the changes observed in the fungal community structure in our study could be attributed to the fact that since oxygen would have run out in the Ziplock bags during the storage, the fungal communities changed from predominantly respiratory to fermentative. Hence, Kazachstania and Saccharomyces replaced Wickerhamomyces as the most abundant fungi during storage treatment.

Kazachstania and Saccharomyces are recognized as typical acid-tolerant yeast; they grow well at $\mathrm{pH}$ as low as 3.5 [29]. Most species of Kazachstania can assimilate sucrose, and several species have similar growth rates and ethanol yields as Saccharomyces [30]. The dominance of $S$. cerevisiae over other microbial competitors during fermentation has been traditionally ascribed to its high fermentative power and aptitude to cope with the harsh environmental conditions, i.e., high levels of ethanol and organic acids, low $\mathrm{pH}$, scarce oxygen availability, and depletion of certain nutrients [31-33]. However, $K$. humilis optimally grows at $25-30{ }^{\circ} \mathrm{C}$ and does not grow at $37{ }^{\circ} \mathrm{C}$. Therefore, Kazachstania was the top genus at $30{ }^{\circ} \mathrm{C}$, while Saccharomyces was the dominant genus at $40{ }^{\circ} \mathrm{C}$ (Fig. 4). The richness and diversity in the fungal community remained low due to the high sucrose content, acidic $\mathrm{pH}$, and killer toxins.
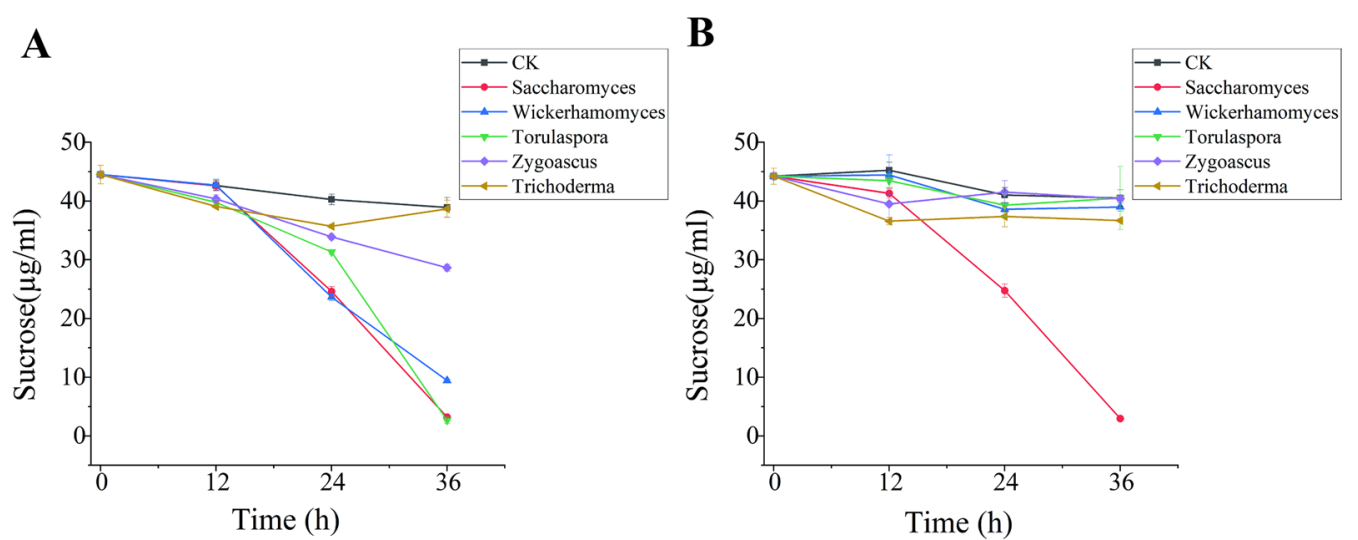

Fig. 7 Sucrose profiles in the sucrose medium of the isolated strains grown at $30^{\circ} \mathrm{C}(\mathbf{A})$ and $40{ }^{\circ} \mathrm{C}(\mathbf{B})$. Data were presented as the mean \pm standard deviation 

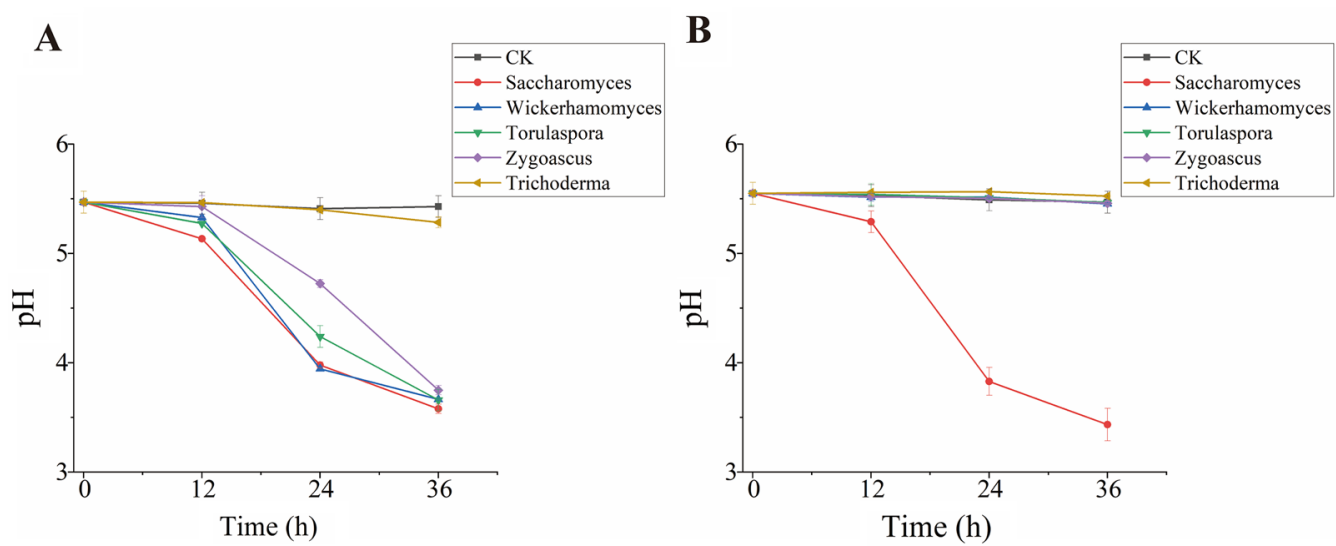

Fig. 8 The pH profiles in the sucrose medium of the isolated strains grown at $30^{\circ} \mathrm{C}(\mathbf{A})$ and $40^{\circ} \mathrm{C}(\mathbf{B})$. Data were presented as the mean $\pm \operatorname{standard}$ deviation

Pre-harvest burnt cane is more vulnerable to opportunistic microbial infection due to stalk rind splitting and loss of wax integrity, causing seepage of juice and an increased level of exposure [34]. The differences in storage temperature could be the primary cause of the variation of core features. Storage time also played a significant role in influencing community structure and abundance. In agreement with our study, temperature was evaluated as a critical factor in shaping the sugar beet microbial community [35]. Similar findings of rapid deterioration were documented in harvested sugarcane [6]. Although less commonly seen in China due to a low prevalence of mechanical harvesting, billeted or chopped cane is known to deteriorate more rapidly than whole stalk cane due to the larger surface area exposed to infection [36]. Average losses in the untreated billets and whole stalk were 0.735 and 0.502 per day, respectively, indicating that deterioration occurred more rapidly and violently near wounds and cut ends [6]. Cutting the sugarcane stalk in harvesting disrupts the plant physiology. The altered balance among the plant functions leads to many undesirable changes in the composition of the stalk. These adverse effects are worsened by the increased duration of the post-harvest storage period between harvest and mill processing and high ambient temperatures [37]. Due to respiration, an increase in temperature within the heap is likely to have significant consequences for the post-harvest stalk rage and juice quality. Approximately $33 \%$ of the energy released in sucrose's oxidative catabolism is captured in ATP chemical bonds [38]. The remaining energy degenerates as heat that significantly contributes to the warming of the storage piles [37, 39]. This study showed that reducing sucrose percent in the harvested and stored sugarcane increased by the cut-to-crush delay and high atmospheric temperature.
Therefore, appropriate measures should be adopted to avoid wounds, cut ends on canes, and complete the crushing in a shorter delay of the harvest-to-crush, keeping ventilation and low temperature as much as possible during transportation, especially for the mechanically chopped cane.

The RDA analysis revealed the $\mathrm{pH}$ as another critical environmental factor. We observed that sucrose reduced by $53.9 \%$ in the first $12 \mathrm{~h}$. pH was also related to sucrose conversion and declined ahead of sucrose. Similar results have been documented by previous studies as well [40]. A rapid decrease in $\mathrm{pH}$ and a rise in titratable acidity during storage increased at higher temperatures [41]. After milling, the acid-producing microorganisms, including Penicillium, Lactobacillus, Leuconostoc, and yeast, increased in the stored sugarcane $[2,12,13]$, which lowered the sucrose content, juice purity, and $\mathrm{pH}$, especially under anaerobic conditions $[5,13]$. The glucose and fructose were converted to organic acids and mannitol by the enzymes secreted by these microorganisms, which resulted in more deleterious than the simple loss of sucrose [36]. Since pH is very easy to measure, it can be used as a suitable physio-biochemical indicator of postharvest deterioration of sugarcane.

\section{Conclusions}

Microecology change is considered to be an essential factor of crop deterioration after harvest. This study was conducted to investigate the variation in fungal communities and changes in relevant environmental factors in the post-harvest deterioration of sugarcane. The very low richness and diversity primarily dominated by yeast were observed in the fungal community during the cane storage. Wickerhamomyces (W. anomalus), Kazachstania (K. humilis), and Saccharomyces (S. 
cerevisiae) were the most abundant fungal genera (species) in the deteriorating cane. The differences in the storage temperature were observed to be the primary cause of variation of core features. Storage time also played a significant role in shaping the fungal community by deciding the relative abundance. The identified top genera in deteriorating sugarcane could be used as a biomarker.

Pre-harvest burnt cane is not permitted in China now, but billeted or chopped cane is becoming more prevalent in mechanical harvesting due to the higher labor cost. The additional effects associated with largescale stockpiling, mechanical loading, and delivery of cane consignments from the field to the mill were also not considered in this research. For a biomarker to be effective as a post-harvest deterioration indicator, it should become apparent even at low levels of deterioration; it should also be adapted and tested for the type of harvesting system used and the particular type of environmental conditions the cane is exposed. Future work should initially focus on broad-based microbial (fungal and bacterial) investigations under different harvesting and environmental conditions, including a combination of both culture-based and molecular-based analyses, to determine the identities of the microbial populations contributing to the post-harvest deterioration process.

\section{Methods}

\section{Sugarcane preservation after harvest}

A total of 30 healthy sugarcane stalks were sampled from the same field in Fusui, China. Six clean harvested stalks without leaf sheath were surface-sterilized and randomly crushed into bagasse using DM540-CPS. This system rapidly smashed sugarcane with a highspeed rotating blade without pressing or producing cane juice, which the stalks were immediately shredded using DM540 (IRBI Machines \& Equipment Ltd, Brazil), blended, and transmitted by CPS (Cane presentation system, Bruker Optik GmbH, Germany) [42]. The cane bagasse of five groups was packed in 50 zip lock bags (500 g each), 40 randomly separated as two groups stored at $30^{\circ} \mathrm{C}$ and $40^{\circ} \mathrm{C}$ for 4 days. Sampling from each bag was performed at $0 \mathrm{~h}, 12 \mathrm{~h}, 36 \mathrm{~h}, 60 \mathrm{~h}$, and $84 \mathrm{~h}$. A total of $100 \mathrm{~g}$ bagasse was blended in cold $\mathrm{dd}_{2} \mathrm{O}$ using a juicer. The blended solution was filtered through eight sterile bandages and centrifuged at 5,000 rpm for $10 \mathrm{~min}$ at $4{ }^{\circ} \mathrm{C}$. The supernatant was stored at $-20{ }^{\circ} \mathrm{C}$, while the pellet, deemed to have epiphytic stalk microbial communities, was stored at $-80{ }^{\circ} \mathrm{C}$. The bagasse filtrates' $\mathrm{pH}$ was determined using a $\mathrm{pH}$ meter (Seven Compact, Mettler Toledo). There were three biological replicates in each treatment group.

\section{Isolation and functional verification of strains}

The sugarcane juice was diluted and coated on separation plates. After culturing, different colonies were selected according to colony characteristics for pure culture and molecular identification. Three kinds of culturing plates (PDA, YPD, and WL) were used to obtain isolated fungal genera [43-45]. The isolated strains were inoculated in $1 \mathrm{~mL}$ PDW medium, cultured at $30{ }^{\circ} \mathrm{C}$, and $220 \mathrm{rpm}$ for $6 \mathrm{~h}$. Then, $100 \mu \mathrm{l}$ of the fungal solution was inoculated in a $100 \mathrm{~mL}$ sucrose medium, cultured at $30{ }^{\circ} \mathrm{C}$ and $40{ }^{\circ} \mathrm{C}$ for $0 \mathrm{~h}, 12 \mathrm{~h}, 24 \mathrm{~h}$, and $36 \mathrm{~h}$. The culture medium was stored at $-20{ }^{\circ} \mathrm{C}$ to determine sucrose, glucose, fructose contents, and $\mathrm{pH}$. The sucrose medium consisted of $20 \%$ sucrose, $0.1 \%$ glucose, $0.1 \%$ fructose, $1 \% \mathrm{NaCl}$, and $1 \%$ yeast extract by stimulating sugarcane juice composition $[46,47]$. The test was carried out in triplicate.

\section{Soluble carbohydrates quantification by HPAEC-PAD}

Soluble carbohydrates (sucrose, glucose, and fructose) were quantified by high-performance anion-exchange chromatography with pulsed amperometric detection (HPAEC-PAD) in a $40 \mathrm{~mm} \times 250 \mathrm{~mm}$ CarboPac PA-1 column on an ICS 5000 Dionex System (Thermo Scientific, Waltham, MA, USA). Diluted sample solutions (diluted 1000 times in Mili-Q water) were filtered through a $0.22 \mu \mathrm{m}$ membrane before injection, and D-lactose monohydrate (Aladdin D1902045, Shanghai, China) was used as an internal standard. The gradient was established by mixing $60 \%$ eluant $\mathrm{A}\left(\mathrm{H}_{2} \mathrm{O}\right)$ with $40 \%$ eluant B $\left(500 \mathrm{mmol} \mathrm{L}{ }^{-1} \mathrm{NaOH}\right)$ for 5 min, using a flow rate of $1 \mathrm{~mL} \mathrm{~min}^{-1}$ through the column $[48,49]$. Each sample was measured twice for technical replicates.

\section{DNA extraction and sequencing}

The community DNA was extracted by the ALFA-SEQ Soil DNA kit (mCHIP, R0911) and quantified by Qubit 3.0 Fluorometer (Life Technologies, Carlsbad, CA, USA) [50]. For amplicon library construction, we used the ITS2 primer pair [ITS21F (5'-CTTGGTCATTTAGAGGAA GTAA- $3^{\prime}$ ) and ITS22R (5'-GCTGCGTTCTTCATCGAT GC- $\left.3^{\prime}\right)$ ]. The PCR mixture contained $5 \times$ TransStart FastPfu buffer $(4 \mu \mathrm{L}), 2.5 \mathrm{mM}$ dNTPs $(2 \mu \mathrm{L})$, forward primer $(5 \mu \mathrm{M}, 0.8 \mu \mathrm{L})$, reverse primer $(5 \mu \mathrm{M}, 0.8 \mu \mathrm{L})$, TransStart FastPfu DNA Polymerase $(0.4 \mu \mathrm{L})$, template DNA (10 ng), and $\mathrm{dd}_{2} \mathrm{O}$ (up to $20 \mu \mathrm{L}$ ). The PCR program was as follows: $95{ }^{\circ} \mathrm{C}$ for $3 \mathrm{~min}, 35$ cycles at $95{ }^{\circ} \mathrm{C}$ for $30 \mathrm{~s}$, $55{ }^{\circ} \mathrm{C}$ for $30 \mathrm{~s}$, and $72{ }^{\circ} \mathrm{C}$ for $45 \mathrm{~s}$ with a final extension at $72{ }^{\circ} \mathrm{C}$ for $10 \mathrm{~min}$. The PCR products were purified using the AxyPrep DNA Gel Extraction Kit (Axygen Biosciences, Union City, CA, USA). The purified amplicons were pooled in equimolar and paired-end sequenced $(2 \times 300)$ on an Illumina MiSeq platform (Illumina, San 
Diego, USA) by Majorbio Bio-Pharm Technology Co. Ltd., Shanghai, China. There were three biological replicates in each treatment group.

\section{Statistical and bioinformatics analysis}

The $\mathrm{pH}$ and sugar concentration data were processed using Microsoft Excel and Origin 2016 software and analyzed using SAS statistical software (SAS incorporation, USA).

The raw ITS2 gene sequencing reads were quality-filtered using Trimmomatic, Cutadapt (version 1.9.1) was used to identify and remove primer sequences, and pairends were spliced using USEARCH (version 10) [51-53]. Operational Taxonomic Units (OTUs) were clustered at a 97\% similarity cutoff. We plotted and calculated a rarefaction curve on the BMKCloud platform. Alpha diversity indices (Chao 1, Shannon, and Simpson index) were determined through Microbiome Analyst, a web-based tool (https://www.microbiomeanalyst.ca/). Principal Components Analysis (PCA) was performed using the "vegan" package in R v. 3.6.3 and plotted on image GP platform (http://www.ehbio.com/ImageGP). The variance explained by parameters was analyzed using a PERMANOVA test in R software (vegan package v. 3.6.3).

The significant differences in relative abundance (average value of three replicates) of fungal genera among treatment groups were determined by one-way variance analysis $(p \leq 0.05)$. Corrplot package in $\mathrm{R}$ software was used to analyze the correlation between the top fourteen genera and six selected environmental factors (temperature, storage time, sugar content, and $\mathrm{pH}$ ). Further, a redundancy analysis (RDA) was performed to investigate the taxa-environment relationships based on OTUs using the "vegan" package in R v. 3.6.3.

\section{Supplementary Information}

The online version contains supplementary material available at https://doi. org/10.1186/s13068-021-02004-x.

\footnotetext{
Additional file 1: Table S1. Sequence characteristics obtained in stored cane stalks. Table S2. OTU and Sequence number obtained in stored cane stalks. Figure $\mathbf{S} 1$. Sequence rarefaction curve. CK: before storage $(n=3)$; s12h30c: stored $12 \mathrm{~h}$ in $30^{\circ} \mathrm{C}(n=3)$; s12h40c: stored $12 \mathrm{~h}$ in $40^{\circ} \mathrm{C}$ $(n=3)$; s60h30c: stored $60 \mathrm{~h}$ in $30^{\circ} \mathrm{C}(\mathrm{n}=3)$; s60h40c: stored $60 \mathrm{~h}$ in $40^{\circ} \mathrm{C}$ $(n=3)$. Figure $\mathbf{S 2}$. The glucose and fructose profiles in sucrose medium of isolated strains grown at $30^{\circ} \mathrm{C}$ and $40^{\circ} \mathrm{C}$. (a) Glucose at $30^{\circ} \mathrm{C}$; (b) glucose at $40{ }^{\circ} \mathrm{C}$; (c) fructose at $30^{\circ} \mathrm{C}$; (d) fructose at $40{ }^{\circ} \mathrm{C}$. Data are presented as the mean \pm standard deviation. There were three biological replicates in each treatment group. Figure S3. PCR amplification for amplicon library construction using ITS21F/ITS22R primer pair. Lane 1: DNA marker; lanes 2-4 (CK): before storage; lanes $5-7\left(30^{\circ} \mathrm{C} 12 \mathrm{~h}\right)$ : stored $12 \mathrm{~h}$ in $30^{\circ} \mathrm{C}$; lanes $8-10\left(40{ }^{\circ} \mathrm{C} 12 \mathrm{~h}\right)$ : stored $12 \mathrm{~h}$ in $40^{\circ} \mathrm{C}$; lanes $11-13\left(30^{\circ} \mathrm{C} 60 \mathrm{~h}\right)$ : stored $60 \mathrm{~h}$ in $30^{\circ} \mathrm{C}$; lanes $14-16\left(40{ }^{\circ} \mathrm{C} 60 \mathrm{~h}\right)$ : stored $60 \mathrm{~h}$ in $40^{\circ} \mathrm{C}$.
}

Acknowledgements

Not applicable.

\section{Authors' contributions}

NP and ZY completed the major experiment, analyzed the data, and wrote the manuscript. ZW and JH participated in the microbiome analysis and sugar content determination. $M Z$ designed the project, supervised the experiments, interpreted the data, and finalized the manuscript. MTK and BC revised the manuscript. All authors read and approved the final manuscript.

\section{Funding}

This work was funded by the China Agricultural Research System of MFA and MARA (CARS 170190) and the Science and Technology Major Project of Guangxi (AA17202042-7).

\section{Availability of data and materials}

The datasets supporting the conclusions of this article are included within the article and its Additional files.

\section{Declarations}

Ethics approval and consent to participate

Not applicable.

\section{Consent for publication}

Not applicable.

\section{Competing interests}

The authors declare that they have no competing interests.

\section{Author details}

${ }^{1}$ Guangxi Key Laboratory for Sugarcane Biology \& State Key Laboratory for Conservation and Utilization of Subtropical Agro-Bioresources, Guangxi University, Nanning 530005, China. ${ }^{2}$ Sugarcane Biotechnology Group, Nuclear Institute of Agriculture (NIA), Tando Jam, Pakistan.

Received: 3 April 2021 Accepted: 23 June 2021

Published online: 02 July 2021

\section{References}

1. Huang JF, Khan MT, Perecin D, et al. Sugarcane for bioethanol production: potential of bagasse in Chinese perspective. Renew Sustain Energy Rev. 2020;133:110296.

2. Hector S, Willard K, Bauer R, et al. Diverse exopolysaccharide producing bacteria isolated from milled sugarcane: implications for cane spoilage and sucrose yield. PLOS ONE. 2015;10(12):e0145487.

3. Eggleston G. Deterioration of cane juice-sources and indicators. Food Chem. 2002;78(1):95-103.

4. Saxena PS, Sharma RP, Sharma ML. Impact of cut to crush delay and bio-chemical changes in sugarcane. Aust J Crop Sci. 2010;4(9):692-9.

5. Solomon S. Postharvest deterioration of sugarcane. Sugar Tech. 2009;11(2):109-23.

6. Solomon S, Singh P, Shrivastava AK, et al. Physico-chemical method of preserving sucrose in harvested sugarcane at high ambient temperature in a sub-tropical climate. Sugar Tech. 2011;13(1):60-7.

7. Souza RA, Telles TS, Machado W, et al. Effects of sugarcane harvesting with burning on the chemical and microbiological properties of the soil. Appl Soil Ecol. 2012;155(2):1-6.

8. Silva MJD, Neves LDO, Correa MHF, et al. Quality indexes, and performance in mechanized harvesting of sugarcane at a burnt cane and green cane. Sugar Tech. 2021;23(3):499-507.

9. Klammsteiner T, Turan V, Oberegger $S$, et al. Suitability of black soldier fly frass as soil amendment and implication for organic waste hygienization. Agronomy. 2020;10(10):1578.

10. Turan V, Schröder P, Bilen S, et al. Co-inoculation effect of Rhizobium and Achillea millefolium L. oil extracts on growth of common bean (Phaseolus vulgaris L.) and soil microbial-chemical properties. Sci Rep. 2019;9(1):15178.

11. Stevenson JA, Rands RD. An annotated list of the fungi and bacteria associated with sugarcane and its products. Hawaii Plant Rec. 1938;42(4):247-313 
12. Misra V, Mall AK, Shrivastava AK, et al. Assessment of Leuconostoc spp. invasion in standing sugarcane with cracks internode. J Ecol Environ. 2019:40(3):316-21.

13. Misra $\vee$, Mall AK, Pathak AD, et al. Microorganisms affecting postharvest sucrose losses in sugarcane. Int J Curr Microbiol Appl Sci. 2017:6(7):2554-66.

14. Suman A, Solomon S, Yadav DV, et al. Postharvest loss in sugarcane quality due to endophytic microorganisms. Sugar Tech. 2000;2(4):21-5.

15. Foster D. Deterioration of chopped cane. Proc Qld Soc Sugar Cane Technol. 1969;36:21-8.

16. Lionnet GRE, Moodley M. The deterioration of burnt and unburnt cane. Sugar Milling Research Institute, Technical report No. 1682. University of Natal, Durban; 1994. p. 8.

17. Khan MT, Yasmeen S, Khan IA. Comparative analysis of sugarcane genotypes for post-harvest deterioration under natural conditions. Pak J Bot. 2020. https://doi.org/10.30848/PJB2020-4(26).

18. Fredlund E, Druvefors U, Boysen ME, et al. Physiological characteristics of the biocontrol yeast Pichia anomala J121. FEMS Yeast Res. 2002;2(3):395-402.

19. Walker GM. Pichia anomala: cell physiology and biotechnology relative to other yeasts. Antonie Van Leeuwenhoek. 2011;99(1):25-34.

20. Aloui H, Licciardello F, Khwaldia K, et al. Physical properties and antifungal activity of bioactive films containing Wickerhamomyces anomalus killer yeast and their application for preservation of oranges and control of post-harvest green mold caused by Penicillium digitatum. Int J Food Microbiol. 2015;4(200):22-30.

21. Czarnecka M, Żarowska B, Połomska X, et al. Role of biocontrol yeasts Debaryomyces hansenii and Wickerhamomyces anomalus in plants defense mechanisms against Monilinia fructicola in apple fruits. Food Microbiol. 2019;83:1-8.

22. Guo FJ, Ma Y, Xu HM, et al. A novel killer toxin produced by the marinederived yeast Wickerhamomyces anomalus YF07b. Antonie Van Leeuwenhoek. 2013;103(4):737-46.

23. Masih El, Alie I, Paul B. Can the grey mold disease of the grape-vine be controlled by yeast? FEMS Microbiol Lett. 2000;189(2):233-7.

24. Oro L, Feliziani E, Ciani M, et al. Biocontrol of post-harvest brown rot of sweet cherries by Saccharomyces cerevisiae Disva 599, Metschnikowia pulcherrima Disva 267 and Wickerhamomyces anomalus Disva 2 strains. Postharvest Biol Technol. 2014;96:64-8.

25. Oro L, Feliziani E, Ciani M, et al. Volatile organic compounds from Wickerhamomyces anomalus, Metschnikowia pulcherrima, and Saccharomyces cerevisiae inhibits the growth of decay-causing fungi and control postharvest diseases of strawberries. Int J Food Microbiol. 2018;265:18-22.

26. Sun HY, Ke WB, Zhe C, et al. Simultaneous production of single-cell protein and killer toxin by Wickerhamomyces anomalus HN1-2 isolated from mangrove ecosystem. Process Biochem. 2012;47(2):251-6.

27. Padilla B, Gil J, Manzanares P. Challenges of the non-conventional yeast Wickerhamomyces anomalus in winemaking. Fermentation. 2018;4(68):1-14.

28. Fredlund E, Blank LM, Schnürer J, et al. Oxygen- and glucose-dependent regulation of central carbon metabolism in Pichia anomala. Appl Environ Microbiol. 2004;70(10):5905-11.

29. Guerzoni ME, Serrazanetti DI, Vernocchi P, et al. Physiology and biochemistry of sourdough yeasts. In: Handbook on sourdough biotechnology, vol. 1. Boston: Springer; 2013. p. 155-81.

30. Hagman A, Säll T, Compagno C, et al. Yeast "make-accumulate-consume" life strategy evolved as a multi-step process that predates the whole genome duplication. PLoS ONE. 2013;8(7):e68734.

31. Bauer F, Pretorius I. Yeast stress response and fermentation efficiency: how to survive the making of wine. S Afr J Enol Viticult. 2000;21:27-51.

32. Hansen $H$, Nissen $P$, Sommer $P$, et al. The effect of oxygen on the survival of non-Saccharomyces yeasts during mixed culture fermentation of grape juice with Saccharomyces cerevisiae. J Appl Microbiol. 2001;91(3):541-7.
33. Albergaria H, Arneborg N. Dominance of Saccharomyces cerevisiae in alcoholic fermentation processes: role of physiological fitness and microbial interactions. Appl Microbiol Biotechnol. 2016;100(5):2035-46.

34. Trujillo-Narcía A, Rivera-Cruz MC, Magaña-Aquino M, et al. The burning of sugarcane plantations in the tropics modifies the microbial and enzymatic processes in soil and rhizosphere. J Soil Sci Plant Nutr. 2019;19(4):906-19.

35. Liebe S, Wibberg D, Winkler A, et al. Taxonomic analysis of the microbial community in stored sugar beets using high-throughput sequencing of different marker genes. FEMS Microbiol Ecol. 2016;92(2):fiw004.

36. Eggleston G, Morel du Boil PG, Walford SN. A review of sugarcane deterioration in the United States and South Africa. In: Proceedings of the South African sugar technologists' association, vol 81. 2008. pp. 72-85.

37. Verma AK, Singh SB, Agarwala AK, et al. Influence of post-harvest storage temperature, time, and invertase enzyme activity on sucrose and weight loss in sugarcane. Postharvest Biol Technol. 2012;73:14-21.

38. Siedow J, Day D. Respiration and photorespiration. In: Biochemistry and molecular biology of plants. New York: Wiley; 2000. p. 676-728.

39. Campbell LG, Klotz KL. Storage. In: Draycott AP, editor. Sugar beet. Oxford: Blackwell Publishing Ltd.; 2006. p. 387-408.

40. Singh I, Solomon S, Shrivastava AK, et al. Post-harvest quality deterioration of cane juice: physio-biochemical indicators. Sugar Tech. 2006;8(2):128-31.

41. Mao L, Que F, Wang G. Sugar metabolism and involvement of enzymes in sugarcane (Saccharum officinarum L.) stems during storage. Food Chem. 2006;98(2):338-42.

42. Li X, Ma F, Liang C, et al. Precise high-throughput online near-infrared spectroscopy assay to determine key cell wall features associated with sugarcane bagasse digestibility. Biotechnol Biofuels. 2021;14(1):123.

43. Cappelli A, Valzano M, Cecarini V, et al. Killer yeasts exert anti-plasmodial activities against the malaria parasite Plasmodium berghei in the vector mosquito Anopheles stephensi and mice. Parasites Vectors. 2019;12(1):329.

44. Li Z, Li H, Song K, et al. Performance of non-Saccharomyces yeasts isolated from Jiaozi in dough fon and steamed bread making. LWT Food Sci Technol. 2019;111:46-54.

45. Sasano Y, Haitani Y, Ohtsu I, et al. Proline accumulation in baker's yeast enhances high-sucrose stress tolerance and fermentation ability in the sweet dough. Int J Food Microbiol. 2012;152(1-2):40-3.

46. Qing LI, Liu HX, Zhou SJ, et al. Analysis and investigation of the component differences of the first and second expressed juice of sugar cane mills. Food Res Dev. 2015;13:5-8.

47. Zheng C. Discussion on the components of sugarcane juice and waste honey in China. Sugarcane Canesugar. 1989;2:40-8.

48. Aldrete-Herrera PI, López MG, Medina-Torres L, et al. Physicochemical composition and apparent degree of polymerization of fructans in five wild agave varieties: potential industrial use. Foods. 2019;8(9):404.

49. Vesna V, Sladana D, Tomislav T, et al. Two aspects of honeydew honey authenticity: application of advance analytical methods and chemometrics. Food Chem. 2020;305(1):1-9.

50. Peng CA, Zhi CA, Qian ZA, et al. Hyperthermophilic composting significantly decreases $\mathrm{N}_{2} \mathrm{O}$ emissions by regulating $\mathrm{N}_{2} \mathrm{O}$-related functional genes. Bioresour Technol. 2019;272:433-41.

51. Bolger AM, Lohse M, Usadel B. Trimmomatic: a flexible trimmer for Illumina sequence data. Bioinformatics. 2014;30(15):2114-20.

52. Martin M. Cutadapt removes adapter sequences from high-throughput sequencing reads. Embnet J. 2011;17(1):10-2.

53. Edgar RC. UPARSE: highly accurate OTU sequences from microbial amplicon reads. Nat Methods. 2013;10(10):996-8.

\section{Publisher's Note}

Springer Nature remains neutral with regard to jurisdictional claims in published maps and institutional affiliations. 